\title{
Nurse-run clinics in primary care increased secondary prevention in coronary artery disease
}

Campbell NC, Ritchie LD, Thain J, et al. Secondary prevention in coronary heart disease: a randomised trial of nurse led clinics in primary care. Heart. 1998 Nov; $80: 447-52$.

\section{Question}

Can clinics run by nurses in primary care improve secondary prevention in patients with coronary artery disease (CAD)?

\section{Design}

Randomized, unblinded, controlled trial with 1-year follow-up.

\section{Setting}

19 general practices in northeastern Scotland.

\section{Patients}

1343 patients $<80$ years of age (about $58 \%$ men) with CAD. Patients who had terminal illness or dementia or who were housebound were excluded. Follow-up was $87 \%$.

\section{Intervention}

673 patients were allocated to attend nurse-run clinics in general practice that included regular follow-up visits (usually every 2 to $6 \mathrm{mo}$ ) for 1 year (intervention group). Nurses reviewed patient symptoms, drug treatment, blood pressure and lipid management, physical activity, dietary fat intake, and smoking; promoted aspirin use; made general practitioner referrals; and suggested behavioral changes if appropriate. 670 patients were allocated to standard care (control group).

\section{Main outcome measures}

Aspirin use, blood pressure and lipid management, physical activity, dietary fat intake, smoking status, and overall secondary prevention (based on a cumu* lative score of 6 secondary prevention components, generated by counting the number of appropriate treatments and behaviors per patient) at baseline and at 1 year.

\section{Main results}

$82 \%$ of patients in the intervention group attended at least 1 nurse-run clinic. Analysis was by intention to treat. Nurse-run clinics increased aspirin use (odds ratio [OR] $3.22,95 \%$ CI 2.15 to $4.80)$ and moderate physical activity (OR 1.67, CI 1.23 to 2.26), improved blood pressure management (OR 5.32, $\mathrm{Cl} 3.01$ to 9.41) and lipid management (OR 3.19, CI 2.39 to 4.26), and decreased dietary fat intake (OR 1.47, Cl 1.10 to 1.96 ) but had no effect on smoking cessation (OR $0.78, \mathrm{Cl} 0.47$ to 1.28). The mean change in the cumulative score of 6 secondary prevention components between baseline and 1 year was greater for the intervention group than for the control group $(0.59$ v $0.06, P<0.001)$, which showed an overall improvement by secondary prevention.

\section{Conclusion}

In patients with coronary artery disease, murse-run clinics in primary care that offered medical and lifestyle counseling were effective in secondary prevention. Sources of funding: Chief Scientist Office at the Scottisb Office and Grampian Henthatre Tww: For correspondence: Dr N.C. Campbell, Den partment of General Practice and Pimaly Care, Foresterbill Health Centre, Westhur: Road, Aberdeen AB25 2AY, Sootlind, UK $F A X$ 44-1224-840791.

Abstract and Commentary also published if ACP Joumal Chb. 1999;130:80.

\section{Commentary}

The studies by Campbell and Cline and their colleagues build on growing evidence that secondary prevention of chronic disease can be enhanced by nursing support to educate patients and help them to follow recommended treatments.

Campbell and colleagues showed that patient counseling (mean $82 \mathrm{~min} / \mathrm{patient}-\mathrm{y}$ ) in nurse-run clinics improved adherence to recommended practices for patients with established CAD, especially for such outcomes as aspirin use, bypertension, and lipid management. Intervention had the greatest benefit for behavior related to lipid management. The finding of suboptimal adherence to cholesterol guidelines is consistent with various other studies that included both generalist and specialist physicians (1). Since 1994, there has been increasing evidence supporting aggressive control of dyslipidemia in patients with existing CAD, with beneits usually apparent after 1 year (2).

Lifestyle variables, in particular tobacco use, were less likely to be influenced by nurse-mediated follow up. The persistence of smoking in $17 \%$ of patients is consistent with findings of resistance to change in other studies (3). As small-scale educational interventions are unlikely to permanently effect change in this group of persons who persistently smoked, more intensive and costly strategies (both behavioral modification and pharmaceutical) need to be considered.

Campbell and colleagues concluded that nurse-run clinics reduced the risk for recurrent $\mathrm{CAD}$. They did not measure cardiovascular events or mortality but suggested that these outcomes could be reduced by up to 1 third. Because of the high risk for patients with established CAD, the benefit of such improvement in mul- tiple risk factors, even of smaller magnitude, is likely to resuit in clinically important decreases in subsequent $\mathrm{CAD}$ events. The presence of multiple risk factors, especially in patients with existing disease, demands more aggressive treatment approach by all providers.

Cline and colleagues followed an elderly group of patients with CHF, initially idertified during hospital admission. Nurse-n education sessions provided > 120 minttes per patient and telephone access. The in tervention group showed an increased tinn to first readmission and trends of refuced mean number of readmissions and days in the hospital. Survival benefits were only parent early in this 1-year trial. Benefits of such a short-term intervention may lesetn over time, given the natural progression of $\mathrm{CHF}$ and the nature of patent complianc' (continued on page 95) 